\title{
Role of green silver nanoparticles synthesized from Symphytum officinale leaf extract in protection against UVB-induced photoaging
}

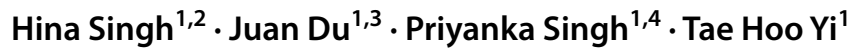

Received: 8 July 2018 / Accepted: 29 August 2018 / Published online: 3 September 2018

(c) The Author(s) 2018

\section{Abstract}

The present study demonstrated the simple, cheap, eco-friendly synthesis of the silver nanoparticles (S-AgNPs) using Symphytum officinale leaf extract. The biosynthesized S-AgNPs were characterized by UV-Vis, FE-TEM, elemental mapping, EDX, zeta potential, XRD, SAED, and FT-IR. The characterization results revealed the irregular shape and relatively stable nature of synthesized S-AgNPs. The average particle size was determined to be $87.46 \mathrm{~nm}$. The zeta potential shows the negative surface charge $(-25.5 \mathrm{mV})$ of S-AgNPs. After characterization, we investigated the anti-aging effect of S-AgNPs in HaCaT keratinocyte cells. HaCaT keratinocyte cells were treated with S-AgNPs at concentrations 1, 10, $100 \mu \mathrm{gL}^{-1}$ after UVB or non-UVB irradiation. The S-AgNPs significantly inhibited the production of matrix metalloproteinase-1 and IL-6 but increased the expression of procollagen type 1. The data suggest that S-AgNPs have photoprotective properties and may have potential to be used as an agent against photoaging.

\section{Graphical abstract}

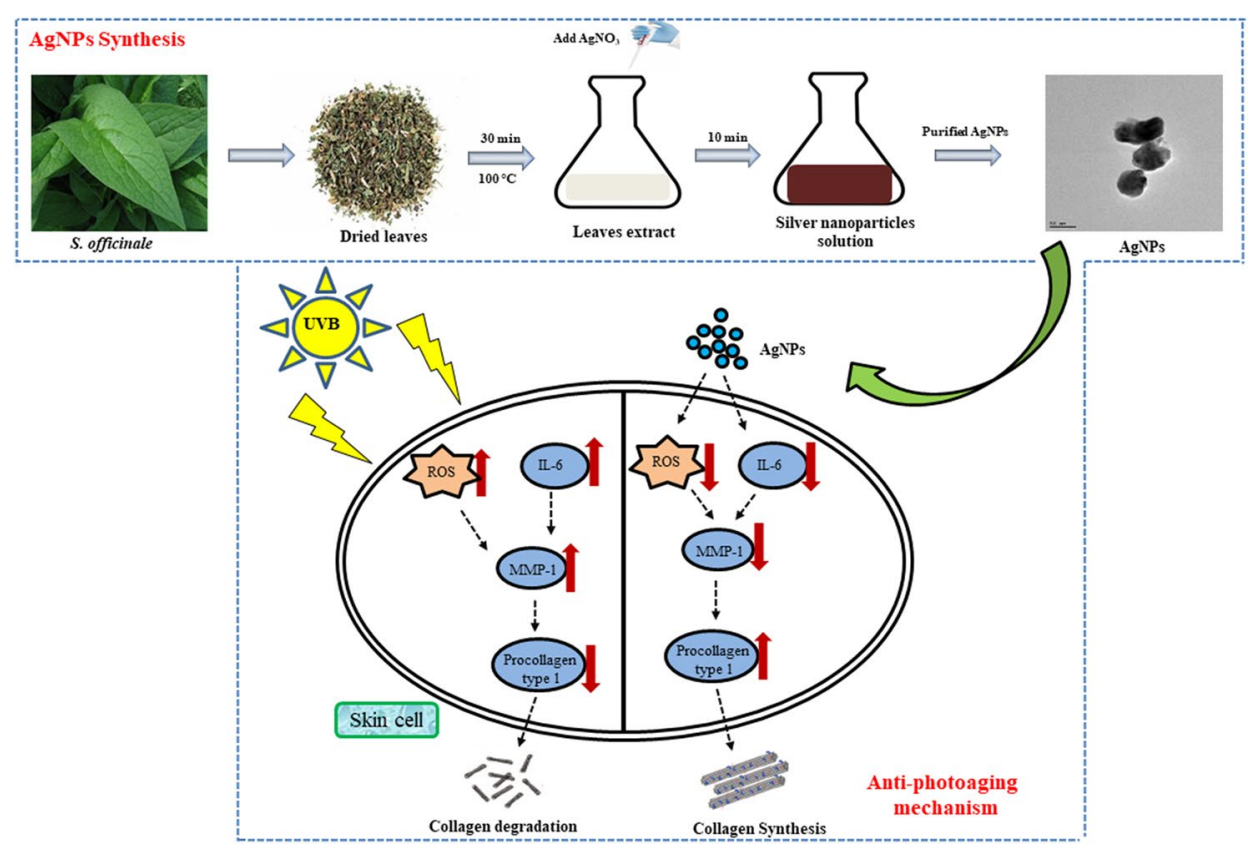

Electronic supplementary material The online version of this article (https://doi.org/10.1007/s40097-018-0281-6) contains supplementary material, which is available to authorized users.

Extended author information available on the last page of the article 
Keywords Symphytum officinale $\cdot$ Silver nanoparticles $\cdot$ Photoaging $\cdot$ MMP-1 $\cdot$ IL-6

\section{Abbreviations}

$\begin{array}{ll}\text { S-AgNPs } & \text { Symphytum silver nanoparticles } \\ \text { UV-Vis } & \text { Ultraviolet-visible spectrophotometer } \\ \text { FE-TEM } & \text { Field emission transmission electron } \\ & \text { microscopy } \\ \text { EDX } & \text { Energy-dispersive X-ray spectroscopy } \\ \text { XRD } & \text { X-ray diffractometer } \\ \text { SAED } & \text { Selected area electron diffraction } \\ \text { FT-IR } & \text { Fourier transform infrared spectroscopy } \\ \text { UVB } & \text { Ultraviolet B } \\ \text { MMP-1 } & \text { Matrix metalloproteinase-1 } \\ \text { IL-6 } & \text { Interleukin 6 } \\ \text { TGF- } \beta & \text { Transforming growth factor beta } \\ \text { S. officinale } & \text { Symphytum officinale } \\ \text { DMSO } & \text { Dimethyl sulfoxide } \\ \text { DMEM } & \text { Dulbecco's modified eagle's medium } \\ \text { FBS } & \text { Fetal bovine serum } \\ \text { ELISA } & \text { Enzyme-linked immune sorbent assay } \\ \text { RT-PCR } & \text { Reverse transcription polymerase chain } \\ & \text { reaction } \\ \text { GAPDH } & \text { Glyceraldehyde 3-phosphate dehydrogenase } \\ \text { ANOVA } & \text { Analysis of variance } \\ \text { SPR } & \text { Surface plasmon resonance } \\ \text { DLS } & \text { Dynamic light scattering } \\ \text { DPPH } & \text { Diphenyl-1-picrylhydrazyl } \\ \text { ROS } & \text { Reactive oxygen species } \\ \text { MTT } & \text { 3-(4,5-Dimethylthiazol-2-yl)-2,5-diphe- } \\ \text { ECM } & \text { nyltetrazolium bromide } \\ & \text { Extracellular matrix } \\ & \end{array}$

\section{Introduction}

Metal nanoparticles exhibit unique chemical and physical properties including large surface/volume ratio, which is useful in different fields such as electronics, photonics, biomedical, catalysis, etc [1]. Among the various noble metals, silver is the metal of the first choice due to their diverse properties especially high antimicrobial and catalytic nature [2,3]. Many standard approaches by means of physical and chemical have been used for the preparation of silver nanoparticles (AgNPs) by several researchers. Generally, conventional physical and chemical methods seem to be very expensive and hazardous [4]. Hence, there is always a need for developing an eco-friendly process for the synthesis of nanoparticles which does not use any harmful or toxic agents. The most recent environment-friendly approach is using green chemistry technology [5]. The green synthesis approach provides most advantages over the chemical and physical method as it is fast, cost-effective, eco-friendly and easy to scale up for large-scale synthesis without applying high energy, high pressure, high temperature and toxic chemicals [6]. The green synthesis approach usually employs microorganism or plant parts for the synthesis of nanoparticles. Recently, many researchers have employed different plant parts such as root, stem, bark, leaf, fruit, bud, and latex for the synthesis of AgNPs [7-10]. Reports have suggested that nanoparticles synthesized from medicinal plants have been found to be pharmacologically active and stable, but no hazardous by-products or toxic chemicals are used in their synthesis [11]. Therefore, as a green approach for the first time, we report the use of Symphytum officinale (Comfrey) leaf extract for the synthesis of AgNPs and further investigating their role in protection against UVBinduced photoaging.

Skin is the primary barrier to the body and protects the body from all kinds of external damage, such as microbial invasion, toxic materials, and ultraviolet (UV) radiation. The solar UV radiation composed of different types of waves depending on the wavelength, UVA (320-400 nm), UVB $(280-320 \mathrm{~nm})$ and UVC (100-280 nm). Among all, UVB is responsible for most of this damage [12]. Acute exposure of human skin to UV irradiation causes sunburn, altered pigmentation, inflammation, immune suppression, and dermal connective tissue damage. It is also reported that chronic exposure to UV irradiation over many years disrupts normal architecture of the skin and ultimately causes premature skin aging (photoaging) due to the degradation of collagen and elastin that normally keep skin firm [13].

Collagen I plays an important role in maintaining skin structure in aged skin cells [12]. The synthesis of collagen I is known to require precursor procollagen type I synthesis by TGF- $\beta /$ Smad signaling in fibroblasts [14]. Contrarily, collagen I can be degraded by UVB-induced ROS activation through two upregulated pathways using the degrading enzymes of collagen I (Matrix metalloproteinases, MMPs) and a collagen synthesis inhibitor (IL-6), thereby leading to the activation of activator protein 1 (AP-1) [13]. Accordingly, the most common feature associated with UVinduced photoaged skin is increased MMP-1 expression and decreased procollagen type I level. Therefore, compounds that decrease the production of MMP-1 and increase the synthesis of procollagen type I may contribute to the prevention of skin photoaging. Various medicinal plant extracts have already been reported to protect the UVB-induced photoaging [12-16]. In the present study, we investigated the role of AgNPs synthesized from $S$. officinale, in protection against UVB-induced photoaging.

Symphytum officinale is a medicinal plant which belongs to family Boraginaceae, commonly known as comfrey. It is a 
perennial plant found in Asia, Europe, and North America. $S$. officinale has been commonly used in folk medicine for the treatment of diarrhea, bronchitis, tuberculosis, ulcers, and hemorrhoids. The plant extract was reported to be used as an ointment to promote the wound healing, reduce the inflammation, for the treatment of broken bones, tendon damages, painful joints, and muscles [17]. Although Comfrey also contains dehydropyrrolizidine alkaloids (DHPAs) because of which its internal application is not recommended [18]. Recently, the root extract of comfrey has shown to have antioxidant and proliferative activity [19]. However, there is no report of AgNPs synthesized from comfrey on UVB-induced photodamage. Here, we hypothesize that the AgNPs synthesized from Comfrey leaf extract could be used for protection against UVB-induced skin photoaging. To test this hypothesis, markers of skin photoaging, MMP-1, IL-6 and procollagen type 1 , were analyzed using PCR with human keratinocyte cells (HaCaT).

\section{Materials and methods}

\section{Material}

Silver nitrate $\left(\mathrm{AgNO}_{3}\right)$, crystal violet, 3-(4,5-dimethylthiazol-2-yl)-2,5-diphenyltetrazolium bromide (MTT), and dimethyl sulphoxide (DMSO) were purchased from SigmaAldrich chemicals (St. Louis, MO). Dulbecco's modified Eagle's medium (DMEM), fetal bovine serum (FBS), and penicillin/streptomycin were purchased from Gibco BRL (Grand Island, NY, USA). ELISA kits for MMP-1 and IL-6 were purchased from R\&D Systems (R\&D Systems Inc., Minneapolis, MN, USA). All other chemicals were of reagent grade. Organic solvents were purchased from Samchun chemicals (Korea). Inorganic salts were purchased from Sigma-Aldrich (St. Louis, MO, USA). Unless otherwise mentioned, solvents were purchased from Samchun chemicals (Seoul, Korea). In the present study, a total of seven plant extracts (Table S1) have been used to synthesize AgNPs. The selection of these plants was depending on the medicinal importance and previous study that has been performed. Dried plants were purchased from mountain rose herbs (Eugene, Oregon, USA).

\section{Preparation of aqueous leaf extract}

$10 \mathrm{~g}$ of the dried plants (root, leaf, seeds) was mixed with $100 \mathrm{~mL}$ of deionized water and autoclaved for $30 \mathrm{~min}$ at $100{ }^{\circ} \mathrm{C}$. The aqueous extract was subsequently centrifuged at $10,000 \mathrm{rpm}$ for $10 \mathrm{~min}$ to remove the debris. Finally, the supernatant obtained was filtered through a $0.45 \mu \mathrm{m}$ PVDF syringe filter (SmartPor, Seoul, Korea). This plant extracts were used for further AgNP synthesis. In the present study, all the plant used for the synthesis of AgNPs are listed in Table S1.

\section{Synthesis of silver nanoparticles}

The AgNPs were synthesized as reported previously [20]. Briefly, aqueous plant extracts were diluted in water to $1: 5$ $(\mathrm{v} / \mathrm{v})$, to this solution the final concentration of $1 \mathrm{mM}$ filtersterilized solution of $\mathrm{AgNO}_{3}$ (Sigma-Aldrich chemicals, St. Louis, MO) has been added. The reaction mixture was kept at $65{ }^{\circ} \mathrm{C}$. The synthesis was monitored for a change in the color, which indicated the synthesis of AgNPs. The AgNPs were collected by high-speed centrifugation at 20,000 rpm for $10 \mathrm{~min}$. The obtained pellet was washed three times with distilled water to remove the unconverted metal ions or any other constituents.

\section{Characterization of silver nanoparticles}

UV-Vis spectrophotometer (UV-Vis) (Optizen POP; Mecasys; Daejeon, Korea) was used to confirm the reduction of metal ions and was scanned in the range of $300-800 \mathrm{~nm}$. The transmission electron microscopy (TEM), elemental mapping, energy-dispersive X-ray spectroscopy (EDX) and selected area electron diffraction (SAED) analysis was performed through field emission transmission electron microscopy (FE-TEM), with a JEM-2100F (JEOL, Tokyo, Japan) instrument operated at $200 \mathrm{kV}$. The sample for FE-TEM was prepared by placing a drop of collected nanoparticles on the carbon-coated copper grid and subsequently drying it at room temperature before transferring it to the microscope. The hydrodynamic size and zeta potential for AgNPs were measured in triplicate using Zetasizer Nano ZS90 (Malvern Instruments, UK), double-distilled water (DDW) was used as a dispersive medium. For DLS and zeta potential analysis, the samples were suspended in water and then used. The $\mathrm{X}$-ray diffraction (XRD) analysis was performed on X-ray diffractometer, D8 Advance, (Bruker), Germany, operated at $40 \mathrm{kV}, 40 \mathrm{~mA}$, with $\mathrm{CuK} \alpha$ radiation, at a scanning rate of $6^{\circ} \mathrm{min}^{-1}$, step size 0.02 , over the $2 \theta$ range of $20^{\circ}-80^{\circ}$. The functional groups capped on the surface of AgNPs were identified using a Fourier transform infrared (FT-IR) spectroscopy (Spectrum One System, Perkin-Elmer, Waltham, MA). For XRD and FT-IR, the purified nanoparticles were dried and obtained in powder form then used.

\section{Antioxidant activity}

Free-radical-scavenging activity was measured by diphenyl1-picrylhydrazyl (DPPH) assay as described previously [21]. A $20 \mu \mathrm{L}$ sample in distilled water was placed in a 96-well plate and $180 \mu \mathrm{L}$ of 2, 2-diphenyl-1-picrylhydrazyl $(0.2 \mathrm{mM})$ in methanol was also added. After a 30-min reaction in dark 
conditions, the absorbance was recorded by a microplate reader (Molecular Devices E09090; San Francisco, CA, USA) at the wavelength of $520 \mathrm{~nm}$. AgNPs synthesized from different plant extracts were tested at the different concentration from 100, 500 and $1000 \mu \mathrm{g} \mathrm{mL} \mathrm{mL}^{-1}$ Arbutin was introduced as a positive control.

\section{Cell culture, UVB irradiation and sample treatment}

HaCaT cells originated from human epidermal keratinocytes (Sciencell, Carlsbad, CA, USA) were cultured in DMEM supplemented with $10 \%$ heat-inactivated FBS and $1 \%$ penicillin-streptomycin at $37^{\circ} \mathrm{C}$ in an atmosphere containing 5\% $\mathrm{CO}_{2}$. When the cells reached more than $80 \%$ confluence, the cells sub-cultured in $35 \mathrm{~mm}$ culture dishes $\left(1.0 \times 10^{5}\right.$ cells $)$ and were rinsed twice with phosphate-buffered saline (PBS). Then, the cells were irradiated with UVB $\left(144 \mathrm{~mJ} \mathrm{~cm}{ }^{-2}\right)$ using UVB radiation machine (Bio-Link BLX-312; Vilber Lourmat GmbH, France). After UVB irradiation, the experimental group cells treated with AgNPs (1, 10 and $100 \mu \mathrm{g}$ $\mathrm{mL}^{-1}$ ). The normal cells were treated with the same dose of AgNPs without UVB irradiation. The controls were without the treatment of AgNPs.

\section{Determination of cell viability}

To test the effect of AgNPs on the viability of HaCaT cells, MTT assay was carried out. MTT assay was performed as described previously [20]. Briefly, after $24 \mathrm{~h}$ of treatment, MTT at a final concentration of $0.1 \mathrm{mg} \mathrm{mL}^{-1}$ was added and further incubated for $2 \mathrm{~h}$. The supernatants were removed and $1 \mathrm{~mL}$ dimethyl sulfoxide (DMSO) was added to dissolve the formazan crystals. Absorbance was determined on a microplate reader (Molecular Devices Filter Max F5; Sunnyvale, CA, USA) at a wavelength of $570 \mathrm{~nm}$.

\section{Measurement of MMP-1 and IL- 6 production}

The level of MMP-1 and IL-6 in the medium were determined after $24 \mathrm{~h}$ of incubation with AgNPs using ELISA kits according to the manufacturer's instructions. Each experiment was analyzed in triplicate.

\section{Reverse transcription (RT)-PCR}

After $24 \mathrm{~h}$ of treatment, total RNA from $\mathrm{HaCaT}$ cells was isolated using Trizol reagent according to the manufacturer's instructions (Invitrogen Life Technologies, Carlsbad, CA). mRNA expression was determined by real-time PCR using SYBR green master mix in a BioRad CFX Connect RealTime PCR Detection System (BioRad, Hercules, CA). The primer pairs as follows: MMP-1, sense 5'-TGGGAGGCA AGTTGAAAAGC-3', antisense 5'-CATCTGGGCTGCTTC
ATCAC-3'; PIP sense 5'-CACAGACAGCTATGACGTG-C$3^{\prime}$ and antisense 5'-TCAGCAGAGAAGACCACCTG-3'. The PCR condition was carried out with an initial denaturation step at $95{ }^{\circ} \mathrm{C}$ for $5 \mathrm{~min}$, followed by 40 cycles of denaturation at $95^{\circ} \mathrm{C}$ for $5 \mathrm{~s}$. The GAPDH gene was used for internal normalization (GAPDH sense 5'-CCAAGGAGTAAGACC CCTGG-3' and antisense 5'-AGGGGAGATTCAGTGTGG TG-3'). PCR products were separated by $2 \%$ agarose gel electrophoresis with ethidium bromide staining.

\section{Statistical analysis}

The data are presented as mean \pm standard deviation values of three independent experiments. Statistical analysis was performed using one-way ANOVA test. Statistical significance was set at $P<0.05$.

\section{Results and discussion}

\section{Synthesis and characterization of AgNPs}

UV-visible spectroscopy is a valuable technique used to detect the characteristic SPR pattern of metal nanoparticles. First, the AgNP synthesis was confirmed by visual observation with the appearance of color change in the reaction mixture. The color of $S$. officinale extract was changed from pale yellow to light brown in $10 \mathrm{~min}$, which resembles the synthesis of AgNPs, as the particles cause surface plasmon resonance (SPR) due to which light brown color appears in the supernatant. Thus, the appearance of a light brown color in the reaction mixture indicated the formation of AgNPs [22]. In the UV-Vis absorption spectrum, a strong peak appeared at $468 \mathrm{~nm}$ for AgNPs synthesized from S. officinale (S-AgNPs), which is due to the SPR band of AgNPs (Fig. 1a). There is also a small peak appeared at $350 \mathrm{~nm}$. The appearance of two peaks shows that the particles are of different sizes. The peak at $350 \mathrm{~nm}$ is for smaller nanoparticles and on the other hand, the $468 \mathrm{~nm}$ peak is a result of larger size nanoparticles. FE-TEM was used for surface morphology and shapes of S-AgNPs, and the images were presented in Fig. 1b. The TEM analysis revealed the irregular shape of synthesized S-AgNPs. The synthesized S-AgNPs ranged in size of 20-94 $\mathrm{nm}$. This size difference can be due to various factors such as temperature, $\mathrm{pH}$, seed concentration or the reducing agent used. The elemental mapping results of the S-AgNPs indicate the maximum distribution of silver element, suggested that silver was the predominant element in the respective nanoproduct (Fig. 2a, b). The EDX analysis showed the highest peak at $3 \mathrm{keV}$ for S-AgNPs (Fig. 2c). Some additional peak for carbon and copper appeared in the EDX spectrum which is due to the TEM grid used for the study. The number and percentage of 


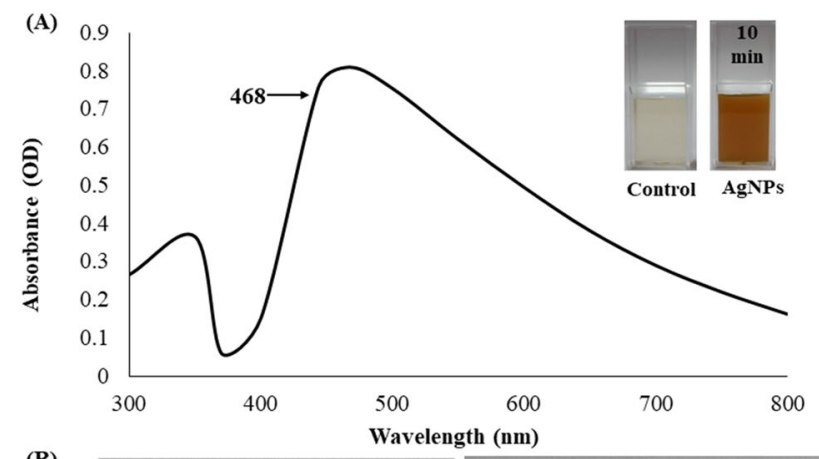

(B)

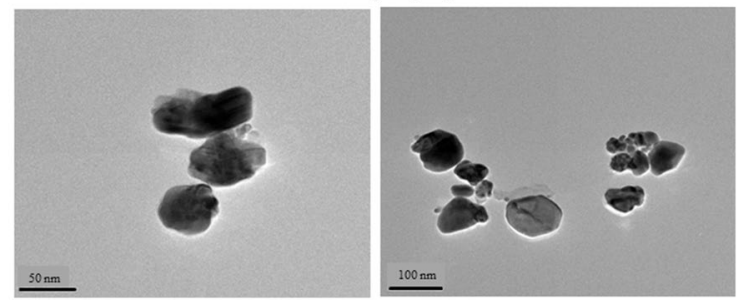

Fig. 1 UV-Vis spectra (a) and FE-TEM images of S-AgNPs (b)
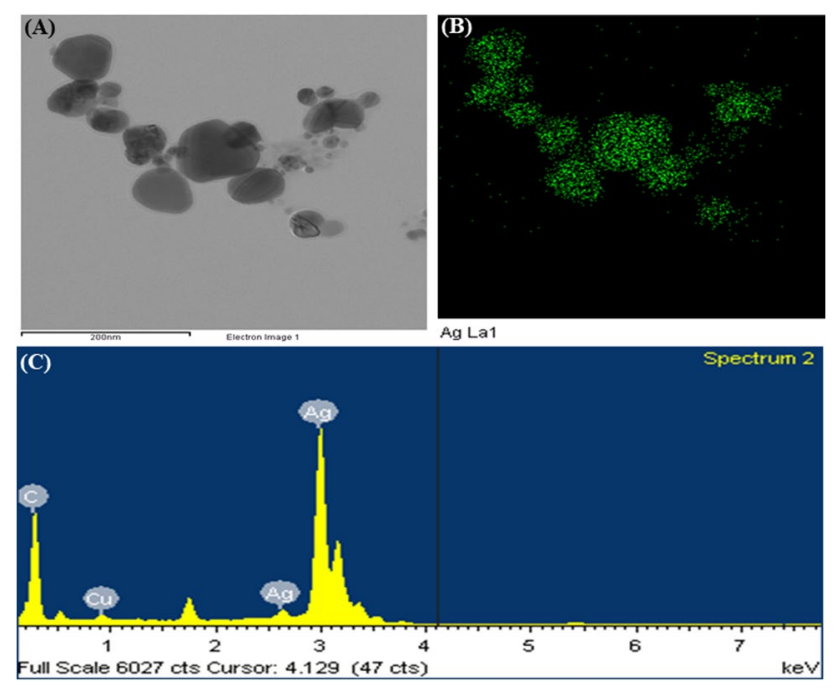

Fig. 2 Elemental mapping result of S-AgNPs, showing distribution of silver in respective nanoproducts $(\mathbf{a}, \mathbf{b})$ and EDX spectrum of S-AgNPs (c)

Table 1 EDAX analysis of S-AgNPs

\begin{tabular}{lll}
\hline Element & Weight (\%) & Atomic (\%) \\
\hline $\mathrm{C} \mathrm{K}$ & 25.91 & 69.74 \\
$\mathrm{Cu} \mathrm{K}$ & 38.50 & 19.59 \\
$\mathrm{Ag} \mathrm{L}$ & 35.59 & 10.67 \\
\hline
\end{tabular}

chemical elements present in EDX spectrum of S-AgNPs are represented in Table 1 . The results are in line with the previously reported work $[22,23]$. The characterization data of

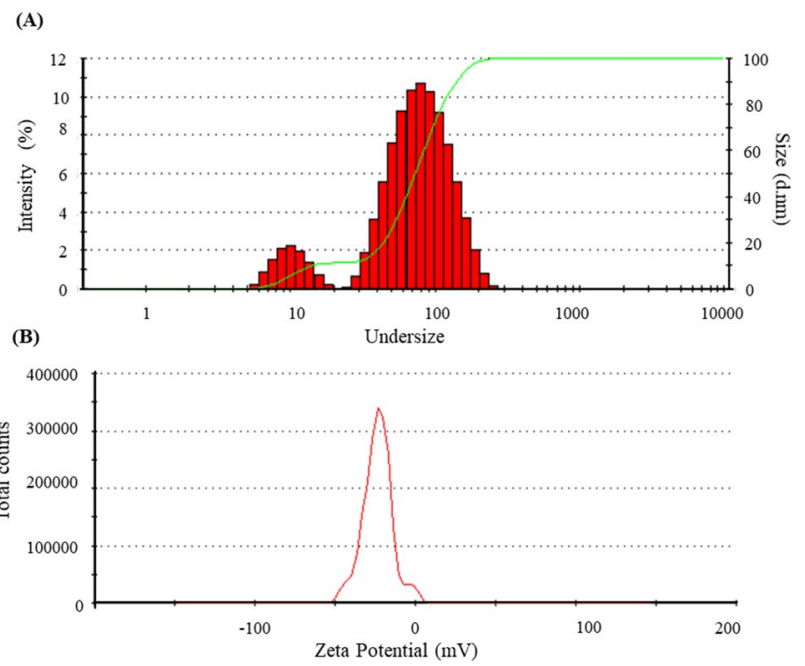

Fig. 3 Particle size distribution (a) and zeta potential result of S-AgNPs (b)

AgNPs synthesized from other six plant extract is shown in supplementary figures (Figs. S1, S2 and S3) The histogram for the particle size distribution of S-AgNPs is depicted in Fig. 3a. The DLS analysis revealed the S-AgNPs with an average diameter of $87.46 \mathrm{~nm}$ in the aqueous colloidal solution. The DLS analysis shows that two peaks of particle dimension is most likely because the produced particles are of different shapes and sizes. The polydispersity index was found to be 0.48 . The zeta potential is used to depict the surface charge and stability of S-AgNPs. As shown in Fig. 3b, the biosynthesized S-AgNPs had a negative charge with a zeta potential value $-25.5 \mathrm{mV}$. This zeta potential value falls within the range of -20 to $-30 \mathrm{mV}$ is considered as moderately stable [24], which clearly indicated that the synthesized S-AgNPs was moderately stable in nature. The X-Ray diffraction pattern of S-AgNPs was shown in Fig. 4a. XRD pattern showed the peaks at 2 values of $38.11^{\circ}, 44.27^{\circ}$, $64.42^{\circ}, 77.47^{\circ}$ and $81.53^{\circ}$ corresponding to the (111), (200), (220), (311) and (222) reflections facets of the cubic crystalline structure. This pattern was like the Braggs's reflection of pure crystalline silver. All these distinct diffraction peaks correspond to the reflection of face-centered cubic ( $f c c$ ) standard silver (JCPDS, PDF\#04-0783), indicated the formation of S-AgNPs. The additional peaks which appeared in XRD are possibly due to the plant bioactive compounds which get coated on to the surface of AgNPs. The XRD result is corresponding to the result in SAED. The appearance of a circular ring in SAED pattern further confirms the crystalline nature of S-AgNPs (Fig. 4b). The findings are in accordance with the previously reported work, wherein green synthesis of S-AgNPs from different plant extract has been reported [20, 21, 23]. 
(A)

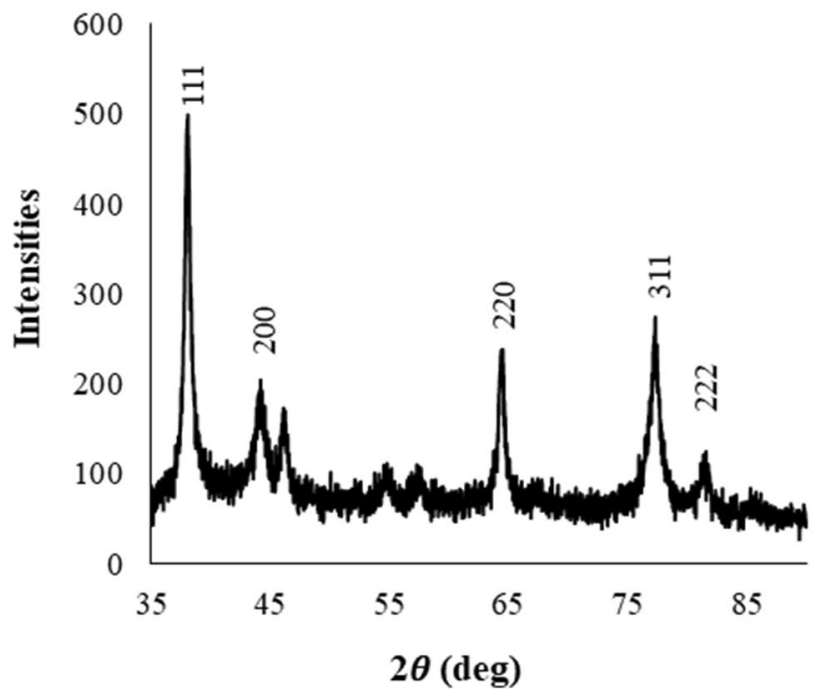

(B)

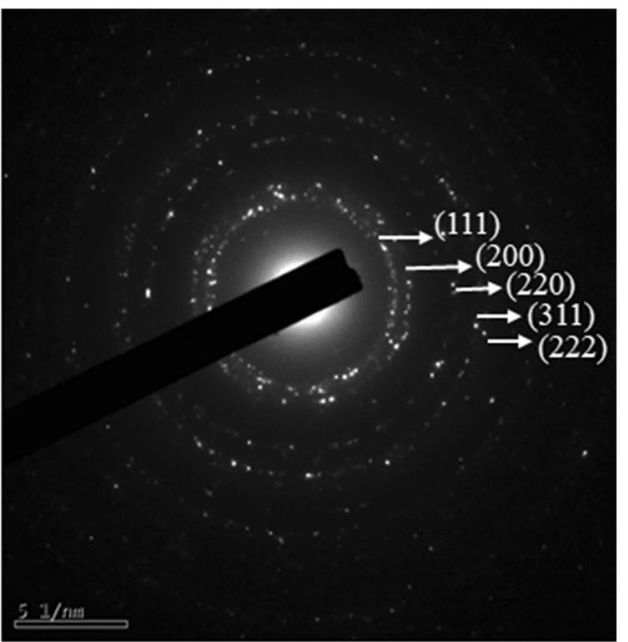

Fig. 4 XRD pattern (a) and SAED pattern of S-AgNPs (b)

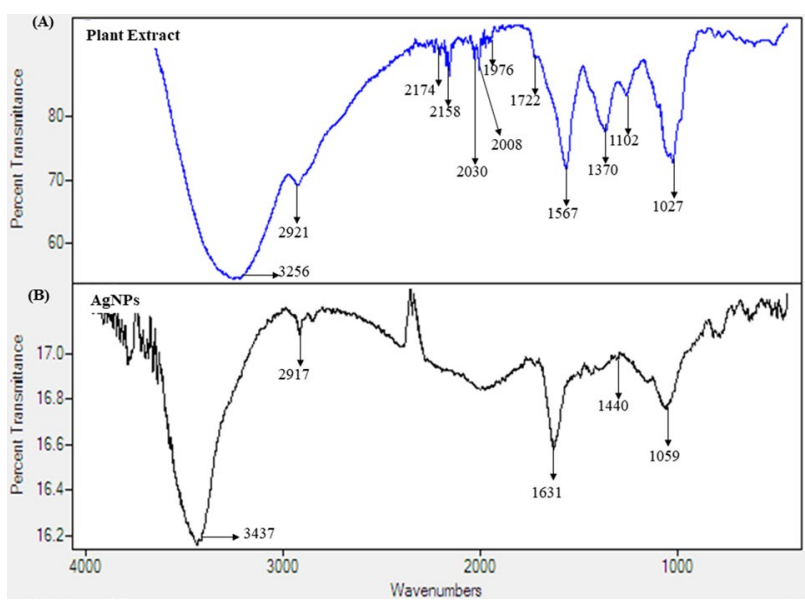

Fig. 5 FTIR spectra of Symphytum officinale leaf extract (a) and S-AgNPs (b)

FT-IR spectroscopy is used for characterizing chemical compounds involved in the formation of S-AgNPs. For the analysis, plant extract and S-AgNPs were used in powdered form. The spectra of plant extract and S-AgNPs were similar (Fig. 5). The peak at $3256 \mathrm{~cm}^{-1}$ and $3437 \mathrm{~cm}^{-1}$ is due to $\mathrm{O}-\mathrm{H}$ vibrations. The other peak at $2921 \mathrm{~cm}^{-1}$ and $2917 \mathrm{~cm}^{-1}$ is a result of $\mathrm{C}-\mathrm{H}$ stretching. In the case of plant extract, some additional peaks appeared in the region of $2000-2200 \mathrm{~cm}^{-1}$, all of them representing $\mathrm{C}-\mathrm{H}$ stretching vibration. Peaks at $1722 \mathrm{~cm}^{-1}$ and $1613 \mathrm{~cm}^{-1}$ corresponds to $\mathrm{C}=\mathrm{O}$ stretching. The bands in the region $1500-1200 \mathrm{~cm}^{-1}$ corresponds to $\mathrm{C}-\mathrm{H}$ stretching vibrations, $\mathrm{N}-\mathrm{H}$ bending, $-\mathrm{CH}_{3}$ wagging and $\mathrm{C}-\mathrm{OH}$ stretching vibrations [25]. $\mathrm{C}-\mathrm{N}$ stretching of amines is at $1027 \mathrm{~cm}^{-1}$ and $1059 \mathrm{~cm}^{-1}$. Peaks at $814 \mathrm{~cm}^{-1}, 820 \mathrm{~cm}^{-1}, 639 \mathrm{~cm}^{-1}$ and $615 \mathrm{~cm}^{-1}$ are due to $\mathrm{C}-\mathrm{H}$ stretching (aromatic) [26].

The peaks at $2174 \mathrm{~cm}^{-1}, 2158 \mathrm{~cm}^{-1}, 2030 \mathrm{~cm}^{-1}$, $2008 \mathrm{~cm}^{-1}, 1976 \mathrm{~cm}^{-1}$ and $1722 \mathrm{~cm}^{-1}$ in comfrey extract were lost intensity in the S-AgNPs, which indicated the involvement of these corresponding groups during the reduction of silver to silver nanoparticles.

\section{Effect of AgNPs on DPPH activity}

UV is the main external factor that causes oxidative stress, such as ROS [27]. ROS simultaneously triggers a series of cascade reactions, eventually leads to overexpression of MMP-1 and degradation of type I procollagen, revealed in photoaging [28]. Thus, DPPH assay was used as a primary method for the selection of AgNPs which can be used for the further anti-aging study. The DPPH will be reduced by accepting the hydrogen or electron, the DPPH reducing ability of AgNPs was measured spectrophotometrically by changing the DPPH color from purple to yellow. Table S2 shows the radical inhibition percentage of all the AgNP samples at different concentrations. Among all the seven plants, AgNP synthesized from S. officinale (S-AgNPs) shown to have DPPH activity as a result S-AgNPs has been selected as a candidate for the further study of anti-aging. The DPPH radical inhibition by S-AgNPs is shown in Fig. 6 . The free-radical inhibition activity of S-AgNPs increased in a dose-dependent manner $\left(100,500\right.$ and $\left.1000 \mu \mathrm{g} \mathrm{mL}^{-1}\right)$. The free-radical scavenging activity of S-AgNPs was not as high as vitamin C (positive control), but S-AgNPs activity can be considered as good. At 100, 500 and $1000 \mu \mathrm{g} \mathrm{mL}{ }^{-1}$, the DPPH inhibition ratio of S-AgNPs were 17.8, 59.5 and 


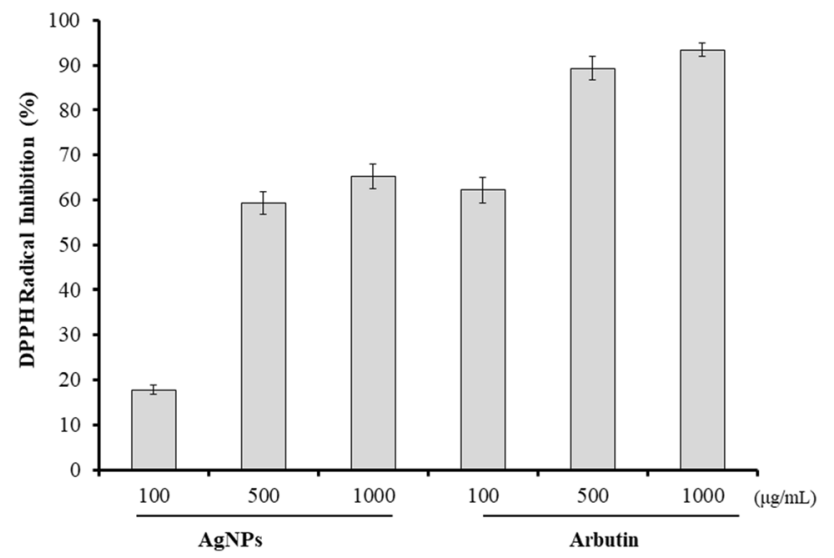

Fig. 6 DPPH free radical scavenging assay of S-AgNPs and arbutin (values are mean \pm SD of three determinations)

$65.2 \%$, respectively. The DPPH inhibition by S-AgNPs can be due to the fact that silver that acts as a good oxidant can easily lose electrons [29]. There is a possibility that the interaction of plant metabolites with metal ions during nanoparticle formation may result in free radical scavenging compounds.

\section{Toxicity analysis of S-AgNPs}

Pharmacological research indicates that solar UV irradiation plays a key role in skin carcinogenesis and photoaging [30]. Chronic solar UV irradiation results in oxidative stress, inflammation, direct and ROS-mediated DNA damage, and dysregulation of cellular signaling pathways, all of which promote skin cancer. Recently, nanoparticles synthesized from the medicinal plant have been widely reported for their anti-inflammatory, anti-cancer, anti-oxidant and antimicrobial effects $[11,31,32]$. There are very few studies showing the effect of nanoparticles on photoaging for e.g., Lee et al. [33] reported the effect of maghemite nanoparticles in protection against ultraviolet-induced photo damage in human skin fibroblasts. Solid lipid nanoparticles of $\mathrm{N}$-6-furfuryl adenine has been reported for the protective effect against photoaging [34]. Here, we hypothesized that S-AgNPs synthesized has photoprotective ability against UVB-induced photoaging. First, MTT analysis was performed to investigate the cytotoxic effects of S-AgNPs on UVB-irradiated and non-UVB-irradiated HaCaT cells. Exposure of cells to UVB caused damage, but this was not statistically significant. S-AgNPs was non-cytotoxic to cells treated with 1, 10 and $100 \mu \mathrm{g} \mathrm{mL}^{-1}$ concentrations (Fig. 7). But in the case of UVB-irradiated cells, the cytotoxicity was observed at the concentration of $100 \mu \mathrm{g} \mathrm{mL}^{-1}$, the cell viability was reduced to $62 \%$. The cell death was due to the UVB exposure. S-AgNPs were found to be non-cytotoxic to HaCaT.

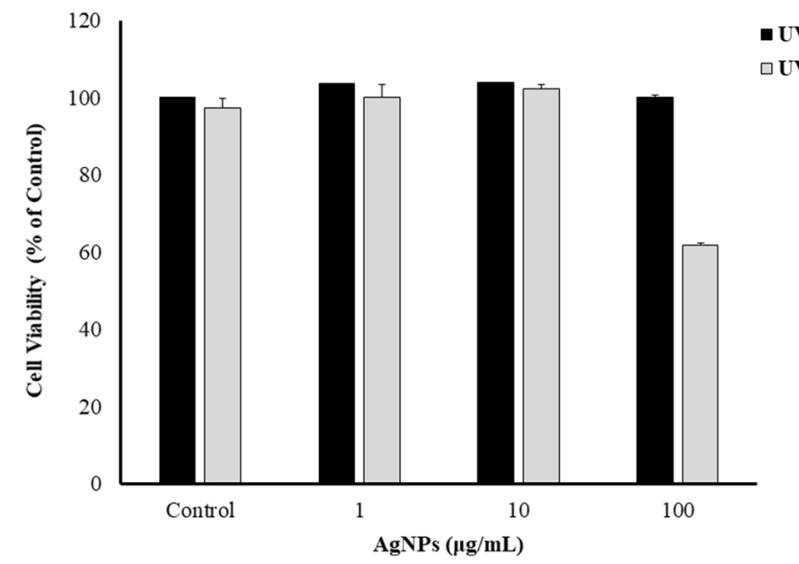

Fig. 7 Effect of S-AgNPs on cell viability of HaCaT cells. HaCaT cells were either irradiated with $100 \mathrm{~mJ} \mathrm{~cm}^{-2}$ or nonirradiated, and then all cells were treated with the indicated concentration of the synthesized S-AgNPs $\left(1,10\right.$ and $\left.100 \mu \mathrm{g} \mathrm{mL}^{-1}\right)$ for $24 \mathrm{~h}$. Data are presented as the mean $\pm \mathrm{SD}$ of three determination

\section{Inhibitory effect of S-AgNPs on the production of MMP-1 and IL-6}

UV stimulated the expression of pro-inflammatory cytokines and epidermal growth factors, which in turn resulted in an increase in the level of MMPs [35]. MMP is a major collagenolytic enzyme responsible for collagen damage in the UVB-irradiated human skin [27]. Reports suggested that collagen damage due to MMP is a major contributor to the photoaging of human skin [36-39]. Therefore, inhibition of MMPs is an important target of treatment in photoaging. We evaluated the effect of S-AgNPs on the secretion of MMP-1 in cultured HaCaT cells. As shown in Fig. 8a, b, UVB exposure led to an abnormal increase in MMP-1 secretion. When treated with S-AgNPs, the level of MMP-1 production started decreasing in a dose-dependent manner. $\mathrm{HaCaT}$ cells treated with 10 and $100 \mu \mathrm{g} \mathrm{mL}^{-1}$ of S-AgNPs had $47.8 \%$ and $53.3 \%$ levels of MMP-1 secretion, respectively. Our result is in line with the previous research which showed the inhibitory effect of maghemite nanoparticles on MMP-1 secretion [33].

Interleukin (IL)-6 is a pro-inflammatory cytokine, which results in overexpression of MMPs and eventually photoaging [15]. We next tested the effect of S-AgNPs on the secretion of IL-6. Our data suggested that UVB irradiation also resulted in a severe increase of the pro-inflammatory cytokine IL-6. After, the treatment with S-AgNPs significantly calmed the activation of the IL- 6 protein, which showed an inhibition rate of $17.8 \%$ and $54.4 \%$ at $10 \mu \mathrm{g} \mathrm{mL}^{-1}$ and $100 \mu \mathrm{g} \mathrm{mL}^{-1}$ (Fig. 8b).

UV irradiation alters the physical properties of skin including loss of type 1 collagen in dermal ECM. Because type I collagen is the main structural protein of dermal ECM, 


\section{(A)}

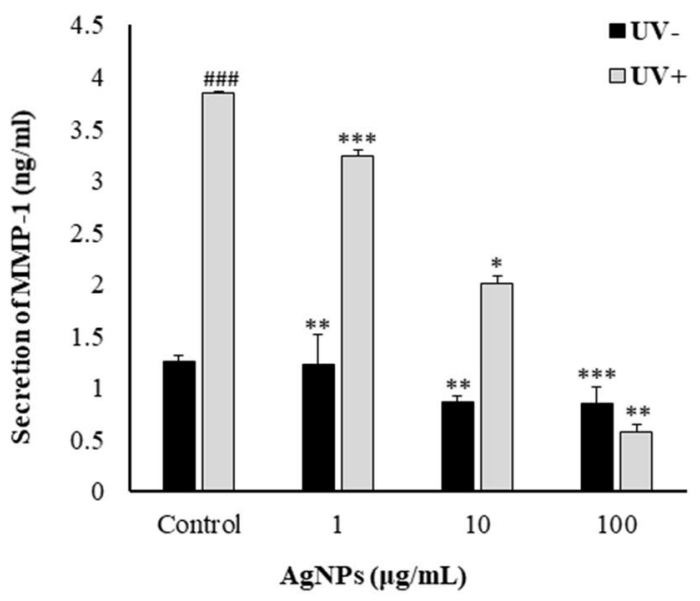

Fig. 8 Effect of S-AgNPs on UVB-induced MMP-1 (a), and IL-6 (b) secretion in $\mathrm{HaCaT}$ cells. $\mathrm{HaCaT}$ cells were either irradiated with $100 \mathrm{~mJ} \mathrm{~cm} \mathrm{~cm}^{-2}$ or nonirradiated, and then all cells were treated with the indicated concentration of S-AgNPs $\left(1,10\right.$ and $\left.100 \mu \mathrm{g} \mathrm{mL}^{-1}\right)$ for $24 \mathrm{~h}$. Data are presented as the calculated percentage of control group

reduction of type I collagen is considered the primary cause of skin aging [27]. In this study, we further investigated the effect of S-AgNPs on MMP-1 and procollagen type $1 \mathrm{mRNA}$ expression in HaCaT cells by RT-PCR analysis. UVB irradiation significantly altered the mRNA levels of MMP-1 and

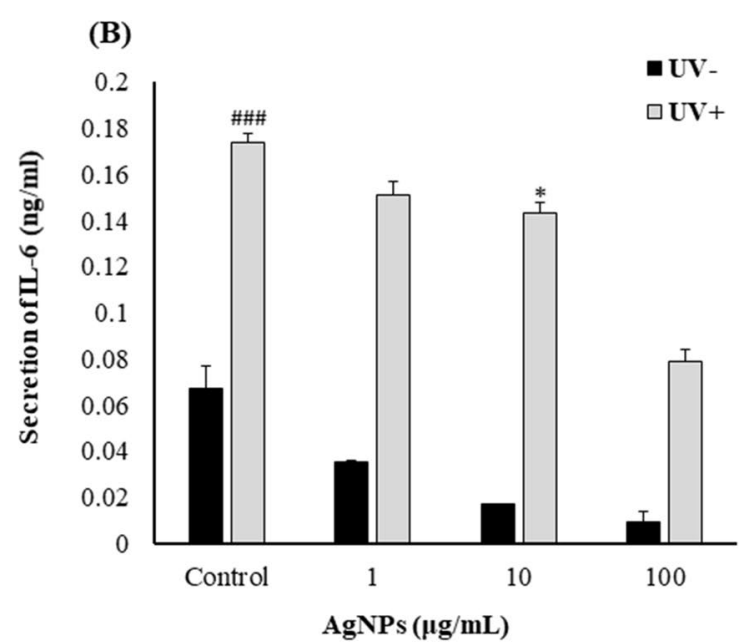

expression and are presented as the mean \pm SD. ${ }^{\# \#} p<0.001$, compared with the nonirradiated group (normal); ${ }^{*} p<0.05$, ${ }^{*} p<0.01$, $* * * p<0.001$, compared with the only ultraviolet B (UVB)-irradiated group (control)

procollagen type 1. As shown in Fig. 9, there was a low basal mRNA expression of MMP-1 in non-UVB-irradiated cells. The MMP-1 mRNA level was significantly elevated in UVBirradiated cells, compared with nonirradiated cells. Consistent with the ELISA result, in the presence of S-AgNPs

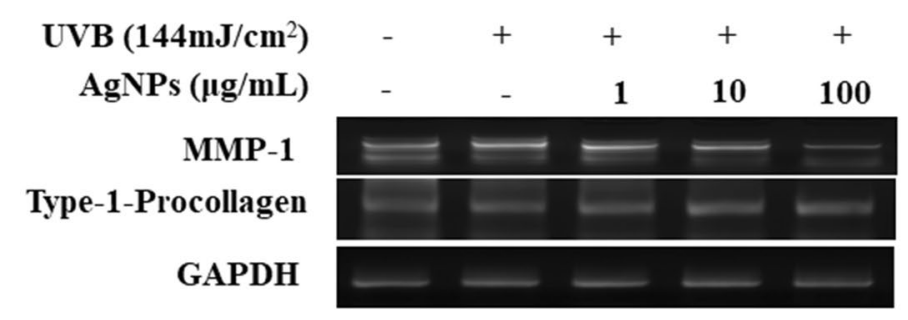

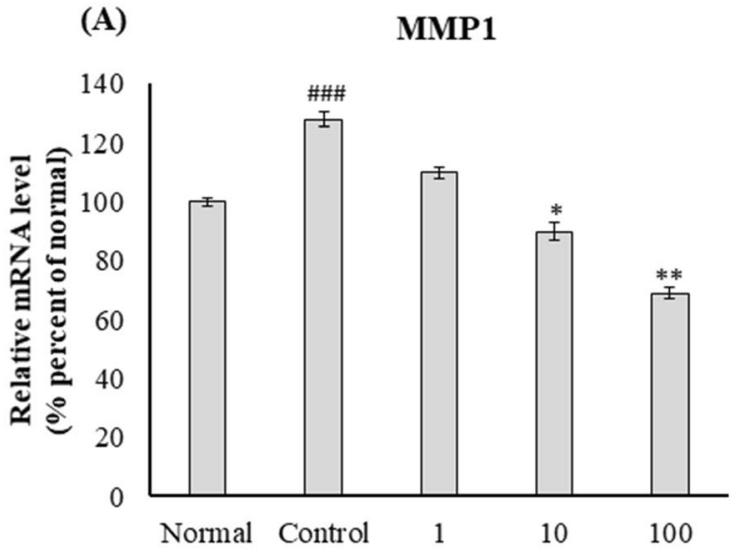

Fig. 9 Effect of AgNPs on MMP-1 (a), and procollagen type 1 (b) mRNA expression. Equimolar quantities of mRNA were quantified relative to GAPDH. Data are presented as the mean \pm SD.

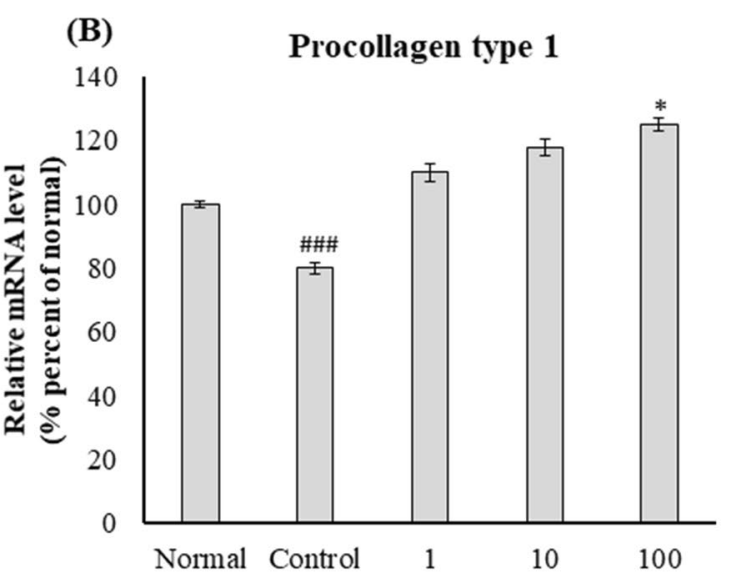

\#\#\# $p<0.001$, compared with the nonirradiated group (normal); $* p<0.05, * * p<0.01$, compared with the only ultraviolet B (UVB)irradiated group (control) 
$\left(100 \mu \mathrm{g} \mathrm{mL}^{-1}\right)$, the MMP-1 expression was decreased to $59 \%$ of the normal value. On the contrary, the treatment with S-AgNPs $\left(1,10\right.$ and $\left.100 \mu \mathrm{g} \mathrm{mL}{ }^{-1}\right)$, promoted the expression of procollagen type I in a dose-dependent manner. Interestingly, our results suggest that S-AgNPs synthesized from comfrey suppressed UVB-induced overexpression MMP-1, IL-6 while enhancing the expression of procollagen type 1 .

\section{Conclusion}

The present study demonstrated the biological synthesis of irregular-shaped S-AgNPs using S. officinale leaf extract. The method was fast, simple, eco-friendly and cheap. Further, the S-AgNPs prevented UVB-induced photoaging in $\mathrm{HaCaT}$ cells. S-AgNPs alleviated the UVB-induced skin damage by suppressing the production of MMP1 and IL-6. On the other hand, S-AgNPs increases the production of procollagen type 1 . These findings suggest that S-AgNPs are a promising agent in protection against photoaging, however, further studies including in vivo needs to be done.

Acknowledgements This work was conducted under the industrial infrastructure program (Grant no. N0000888) for fundamental technologies which is funded by the Ministry of Trade, Industry, and Energy (MOTIE, Korea).

Open Access This article is distributed under the terms of the Creative Commons Attribution 4.0 International License (http://creativeco mmons.org/licenses/by/4.0/), which permits unrestricted use, distribution, and reproduction in any medium, provided you give appropriate credit to the original author(s) and the source, provide a link to the Creative Commons license, and indicate if changes were made.

\section{References}

1. Saha, J., Begum, A., Mukherjee, A., Kumar, S.: A novel green synthesis of silver nanoparticles and their catalytic action in reduction of methylene blue dye. Sustain. Env. Res. 27, 245-250 (2017)

2. Khan, T., Khan, M.A., Nadhman, A.: Synthesis in plants and plant extracts of silver nanoparticles with potent antimicrobial properties: current status and future prospects. Appl. Microbiol. Biotechnol. 99, 9923-9934 (2015)

3. Rostami-Vartooni, A., Nasrollahzadeh, M., Alizadeh, M.: Green synthesis of seashell supported silver nanoparticles using Bunium persicum seeds extract: application of the particles for catalytic reduction of organic dyes. J. Colloid Interface Sci. 470, 268-275 (2016)

4. Zhang, X.F., Liu, Z.G., Shen, W., Gurunathan, S.: Silver nanoparticles: synthesis, characterization, properties, applications, and therapeutic approaches. Int. J. Mol. Sci. 17, 1534 (2016)

5. Song, J.Y., Kim, B.S.: Rapid biological synthesis of silver nanoparticles using plant leaf extracts. Bioprocess Biosyst. Eng. 32, 79 (2009)

6. Albrecht, M.A., Evans, C.W., Raston, C.L.: Green chemistry and the health implications of nanoparticles. Green Chem. 8, 417-432 (2006)
7. Mariselvam, R., Ranjitsingh, A.J., Nanthini, A.U., Kalirajan, K., Padmalatha, C., Selvakumar, P.M.: Green synthesis of silver nanoparticles from the extract of the inflorescence of Cocos nucifera (Family: Arecaceae) for enhanced antibacterial activity. Spectrochim. Part A 129, 537-541 (2014)

8. Danai-Tambhale, S.D., Adhyapak, P.V.: A facile green synthesis of silver nanoparticles using Psoralea corylifolia L. seed extract and their in vitro antimicrobial activities. Int. J. Pharm. Biol. Sci. 5, 457-467 (2014)

9. Chandran, S.P., Chaudhary, M., Pasricha, R., Ahmad, A., Sastry, M.: Synthesis of gold nanotriangles and silver nanoparticles using aloe vera plant extract. Biotechnol. Prog. 22, 577-583 (2006)

10. Gnanajobitha, G., Paulkumar, K., Vanaja, M., Rajeshkumar, S., Malarkodi, C., Annadurai, G., Kannan, C.: Fruit-mediated synthesis of silver nanoparticles using Vitis vinifera and evaluation of their antimicrobial efficacy. J. Nanostructure Chem. 3, 67 (2013)

11. Ahn, S., Singh, P., Jang, M., Kim, Y.J., Castro-Aceituno, V., Simu, S.Y., Kim, Y.J., Yang, D.C.: Gold nanoflowers synthesized using Acanthopanacis cortex extract inhibit inflammatory mediators in LPS-induced RAW264. 7 macrophages via NF- $\kappa$ B and AP-1 pathways. Colloids Surf. B Biointerfaces 160, 423-428 (2017)

12. Sun, Z., Hwang, E., Park, S.Y., Zhang, M., Gao, W., Lin, P., Yi, T.H.: Angelica archangelia prevented collagen degradation by blocking production of matrix metalloproteinases in UVBexposed dermal fibroblasts. Photochem. Photobiol. 92, 604-610 (2016)

13. Ngo, H.T., Hwang, E., Seo, S.A., Park, B., Sun, Z.W., Zhang, M., Shin, Y.K., Yi, T.H.: Topical application of neem leaves prevents wrinkles formation in UVB-exposed hairless mice. J. Photochem. Photobiol. B 169, 161-170 (2017)

14. Quan, T., He, T., Kang, S., Voorhees, J.J., Fisher, G.J.: Solar ultraviolet irradiation reduces collagen in photoaged human skin by blocking transforming growth factor- $\beta$ type II receptor/Smad signaling. Am. J. Pathol. 165, 741-751 (2004)

15. Park, B., Hwang, E., Seo, S.A., Cho, J.G., Yang, J.E., Yi, T.H.: Eucalyptus globulus extract protects against UVB-induced photoaging by enhancing collagen synthesis via regulation of TGF- $\beta$ / Smad signals and attenuation of AP-1. Arch. Biochem. Biophys. 637, 31-39 (2018)

16. Sun, Z., Park, S.Y., Hwang, E., Zhang, M., Seo, S.A., Lin, P., Yi, T.H.: Thymus vulgaris alleviates UVB irradiation induced skin damage via inhibition of MAPK/AP-1 and activation of Nrf2ARE antioxidant system. J. Cell Mol. Med. 21, 336-348 (2017)

17. Staiger, C.: Comfrey: a clinical overview. Phytother. Res. 26, 1441-1448 (2012)

18. Brown, A.W., Stegelmeier, B.L., Colegate, S.M., Gardner, D.R., Panter, K.E., Knoppel, E.L., Hall, J.O.: The comparative toxicity of a reduced, crude comfrey (Symphytum officinale) alkaloid extract and the pure, comfrey-derived pyrrolizidine alkaloids, lycopsamine and intermedine in chicks (Gallus domesticus). J. Appl. Toxicol. 36, 716-725 (2016)

19. Sowa, I., Paduch, R., Strzemski, M., Zielińska, S., Rydzik-Strzemska, E., Sawicki, J., Kocjan, R., Polkowski, J., Matkowski, A., Latalski, M., Wójciak-Kosior, M.: Proliferative and antioxidant activity of Symphytum officinale root extract. Nat. Prod. Res. 32, 605-609 (2018)

20. Singh, H., Du, J., Singh, P., Yi, T.H.: Ecofriendly synthesis of silver and gold nanoparticles by Euphrasia officinalis leaf extract and its biomedical applications. Artif. Cells Nanomed. Biotechnol. 45, 1-8 (2017)

21. Du, J., Singh, H., Yi, T.H.: Antibacterial, anti-biofilm and anticancer potentials of green synthesized silver nanoparticles using benzoin gum (Styrax benzoin) extract. Bioprocess Biosyst Eng. 39, 1923-1931 (2016) 
22. Singh, P., Kim, Y.J., Singh, H., Wang, C., Hwang, K.H., Farh, Mel A., Yang, D.C.: Biosynthesis, characterization, and antimicrobial applications of silver nanoparticles. Int. J. Nanomed. 10, 2567-2577 (2015)

23. Singh, H., Juan, D., Yi, T.H.: Green and rapid synthesis of silver nanoparticles using Borago officinalis leaf extract: anticancer and antibacterial activities. Artif. Cells Nanomed. Biotechnol. 44, 1-7 (2016)

24. Ardani, H.K., Imawan, C., Handayani, W., Djuhana, D., Harmoko, A., Fauzia, V.: Enhancement of the stability of silver nanoparticles synthesized using aqueous extract of Diospyros discolor Wild. leaves using polyvinyl alcohol. InIOP Conf. Ser. Mater. Sci. Eng. 188, 012056 (2017)

25. Bhuyan, B., Paul, A., Paul, B., Dhar, S.S., Dutta, P.: Paederia foetida Linn. promoted biogenic gold and silver nanoparticles: synthesis, characterization, photocatalytic and in vitro efficacy against clinically isolated pathogens. J. Photochem. Photobiol. B 173, 210-215 (2017)

26. Vijayan, R., Joseph, S., Mathew, B.: Indigofera tinctoria leaf extract mediated green synthesis of silver and gold nanoparticles and assessment of their anticancer, antimicrobial, antioxidant and catalytic properties. Artif. Cells Nanomed. Biotechnol. 45, 1-11 (2017)

27. Hwang, E., Park, S.Y., Lee, H.J., Lee, T.Y., Sun, Z.W., Yi, T.H.: Gallic acid regulates skin photoaging in UVB-exposed fibroblast and hairless mice. Phytother. Res. 28, 1778-1788 (2014)

28. Hwang, E.S., Park, S.Y., Lee, H.J., Sun, Z.W., Lee, T.Y., Song, H.G., Shin, H.S., Yi, T.H.: Vigna angularis water extracts protect against ultraviolet b-exposed skin aging in vitro and in vivo. J. Med. Food 17, 1339-1349 (2014)

29. Soshnikova, V., Kim, Y.J., Singh, P., Huo, Y., Markus, J., Ahn, S., Castro-Aceituno, V., Kang, J., Chokkalingam, M., Mathiyalagan, R., Yang, D.C.: Cardamom fruits as a green resource for facile synthesis of gold and silver nanoparticles and their biological applications. Artif. Cells Nanomed. Biotechnol. 45, 1-10 (2017)

30. Sun, Z., Park, S.Y., Hwang, E., Park, B., Seo, S.A., Cho, J.G., Zhang, M., Yi, T.H.: Dietary Foeniculum vulgare Mill extract attenuated UVB irradiation-induced skin photoaging by activating of Nrf2 and inhibiting MAPK pathways. Phytomedicine 23, 1273-1284 (2016)

31. Singh, P., Ahn, S., Kang, J.P., Veronika, S., Huo, Y., Singh, H., Chokkaligam, M., El-Agamy, Farh M., Aceituno, V.C., Kim, Y.J.,

\section{Affiliations}

\section{Hina Singh ${ }^{1,2} \cdot$ Juan Du ${ }^{1,3} \cdot$ Priyanka Sing ${ }^{1,4} \cdot$ Tae Hoo Yi $^{1}$}

Tae Hoo Yi

drhoo@khu.ac.kr

1 Department of Oriental Medicine Biotechnology, College of Life Science, Kyung Hee University Global Campus, 1732 Deokyoungdaero, Giheung-gu, Yongin-si, Gyeonggi-do 446-701, Republic of Korea

2 Present Address: Division of Biomedical Sciences, School of Medicine, University of California, Riverside 92521, USA
Yang, D.C.: In vitro anti-inflammatory activity of spherical silver nanoparticles and monodisperse hexagonal gold nanoparticles by fruit extract of Prunus serrulata: a green synthetic approach. Artif. Cells Nanomed. Biotechnol. 45, 1-11 (2017)

32. Castro-Aceituno, V., Ahn, S., Simu, S.Y., Singh, P., Mathiyalagan, R., Lee, H.A., Yang, D.C.: Anticancer activity of silver nanoparticles from Panax ginseng fresh leaves in human cancer cells. Biomed. Pharmacother. 84, 158-165 (2016)

33. Lee, K.J., An, J.H., Shin, J.S., Kim, D.H., Kim, C., Ozaki, H., Koh, J.G.: Protective effect of maghemite nanoparticles on ultraviolet-induced photo-damage in human skin fibroblasts. Nanotechnology 18, 465201 (2007)

34. Goindi, S., Guleria, A., Aggarwal, N.: Development and evaluation of solid lipid nanoparticles of $N$-6-furfuryl adenine for prevention of photoaging. J. Biomed. Nanotechnol. 11, 1734-1746 (2015)

35. Pillai, S., Oresajo, C., Hayward, J.: Ultraviolet radiation and skin aging: roles of reactive oxygen species, inflammation and protease activation, and strategies for prevention of inflammation-induced matrix degradation-a review. Int. J. Cosmet. Sci. 27, 17-34 (2005)

36. Quan, T., Qin, Z., Xia, W., Shao, Y., Voorhees, J.J., Fisher, G.J.: Matrix-degrading metalloproteinases in photoaging. J. Investig. Dermatol. Symp. Proc. 14, 20-24 (2009)

37. Fisher, G.J., Choi, H.C., Bata-Csorgo, Z., Shao, Y., Datta, S., Wang, Z.Q., Kang, S., Voorhees, J.J.: Ultraviolet irradiation increases matrix metalloproteinase- 8 protein in human skin in vivo. J. Investig. Dermatol. 117, 219-226 (2001)

38. Varani, J., Perone, P., Fligiel, S.E., Fisher, G.J., Voorhees, J.J.: Inhibition of type I procollagen production in photodamage: correlation between presence of high molecular weight collagen fragments and reduced procollagen synthesis. J. Investig. Dermatol. 119, 122-129 (2002)

39. Brennan, M., Bhatti, H., Nerusu, K.C., Bhagavathula, N., Kang, S., Fisher, G.J., Varani, J., Voorhees, J.J.: Matrix metalloproteinase-1 is the major collagenolytic enzyme responsible for collagen damage in UV-irradiated human skin. Photochem. Photobiol. 78, 43-48 (2003)

Publisher's Note Springer Nature remains neutral with regard to jurisdictional claims in published maps and institutional affiliations.
3 Present Address: Henan Province Collaborative Innovation Center for Food Production and Safety, College of Food and Bioengineering, Zhengzhou University of Light Industry, Zhengzhou 450001, China

4 Present Address: DTU Biosustain, Novo Nordisk Foundation Center for Biosustainability, Technical University of Denmark, Lyngby 2800, Denmark 